\title{
Papers
}

\section{Ultrasonic locating devices for central venous cannulation: meta-analysis}

Daniel Hind, Neill Calvert, Richard McWilliams, Andrew Davidson, Suzy Paisley, Catherine Beverley, Steven Thomas

\begin{abstract}
Objectives To assess the evidence for the clinical effectiveness of ultrasound guided central venous cannulation.
\end{abstract}

Data sources 15 electronic bibliographic databases, covering biomedical, science, social science, health economics, and grey literature.

Design Systematic review and meta-analysis of randomised controlled trials.

Populations Patients scheduled for central venous access.

Intervention reviewed Guidance using real time two dimensional ultrasonography or Doppler needles and probes compared with the anatomical landmark method of cannulation.

Data extraction Risk of failed catheter placement (primary outcome), risk of complications from placement, risk of failure on first attempt at placement, number of attempts to successful catheterisation, and time (seconds) to successful catheterisation.

Data synthesis 18 trials (1646 participants) were identified. Compared with the landmark method, real time two dimensional ultrasound guidance for cannulating the internal jugular vein in adults was associated with a significantly lower failure rate both overall (relative risk $0.14,95 \%$ confidence interval 0.06 to 0.33$)$ and on the first attempt $(0.59,0.39$ to 0.88 ). Limited evidence favoured two dimensional ultrasound guidance for subclavian vein and femoral vein procedures in adults $(0.14,0.04$ to 0.57 and 0.29 , 0.07 to 1.21 , respectively). Three studies in infants confirmed a higher success rate with two dimensional ultrasonography for internal jugular procedures $(0.15$, 0.03 to 0.64$)$. Doppler guided cannulation of the internal jugular vein in adults was more successful than the landmark method ( $0.39,0.17$ to 0.92$)$, but the landmark method was more successful for subclavian vein procedures $(1.48,1.03$ to 2.14$)$. No significant difference was found between these techniques for cannulation of the internal jugular vein in infants. An indirect comparison of relative risks suggested that two dimensional ultrasonography would be more successful than Doppler guidance for subclavian vein procedures in adults $(0.09,0.02$ to 0.38$)$.
Conclusions Evidence supports the use of two dimensional ultrasonography for central venous cannulation.

\section{Introduction}

Around 200000 procedures for central venous access are performed in the NHS each year. ${ }^{1}$ Catheters are inserted for several reasons, including haemodynamic monitoring, delivery of blood products and drugs (for example, chemotherapy and antibiotics), haemodialysis, total parenteral nutrition, and management of perioperative fluids. These procedures are performed in a wide range of locations within the hospital and at various insertion sites on the body by medical and, increasingly, nursing staff.

Central venous access is commonly attempted at the internal jugular vein, subclavian vein, femoral vein, or arm veins, using peripherally inserted central catheters. Safe puncture of a central vein (venepuncture) is traditionally achieved by passing the needle along the anticipated line of the vein using anatomical landmarks on the skin's surface (the landmark method). Surgical cut-down is a more invasive and alternative method for gaining central venous access, although it is now less commonly used.

Central venous cannulation can be unsafe: the National Confidential Enquiry into Perioperative Deaths has reported one death resulting from a procedure induced pneumothorax. ${ }^{2}$ Less serious, but still costly for patient discomfort, clinician time, and NHS resources are the varying rates for failure and complications from central venous cannulation. Anomalies in anatomy may cause the operator to pass the needle in an inappropriate direction. The landmark method fails, irrespective of anatomy, if the vein has thrombosed. Each pass of a needle carries the risk of complications, so a successful first attempt is ideal.

The rates, risks, and consequences of complications arising from central venous cannulation vary across patient groups. Infants, obese patients, and those with short necks are more difficult to access. Patients with clotting problems, ventilated patients, and those undergoing emergency pacing procedures may have more serious consequences from a complication associated with venepuncture. ${ }^{2}$ Repeated catheterisation (as in patients requiring chemotherapy or haemodialy-
School of Health and Related

Research

(ScHARR), Regent

Court, Sheffield

S1 4DA

Daniel Hind

research associate

Suzy Paisley

managing director,

ScHARR Rapid

Reviews Group

Catherine Beverley

information officer

Fourth Hurdle

Consulting, London

WC1R 4QA

Neill Calvert

consultant

Royal Liverpool

University Hospital,

Liverpool L7 8XP

Richard

McWilliams

consultant radiologist

Sheffield Teaching

Hospitals NHS

Trust, Royal

Hallamshire

Hospital, Sheffield

S10 2JF

Andrew Davidson

consultant

anaesthetist

Department of

Academic

Radiology,

University of

Sheffield, Northern

General Hospital,

Sheffield S5 7AU

Steven Thomas

senior lecturer

Correspondence to:

D Hind

d.hind@shef.ac.uk

bmj.com 2003;327:361

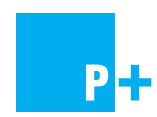

Additional

references appear

on bmi.com 
sis) is a significant risk factor for the formation of thrombus. ${ }^{3}$

Medical ultrasound devices may be used to locate a vein in two ways. Real time ultrasonography generates a two dimensional grey scale image of the vein and surrounding tissues. Continuous wave Doppler ultrasonography generates an audible sound from flowing venous blood, with no information on depth of the vessel. We systematically reviewed randomised controlled trials for evidence of the effectiveness of two dimensional ultrasound guidance and Doppler ultrasound guidance in patients undergoing central venous catheterisation.

\section{Methods}

Our study was commissioned by the National Institute for Clinical Excellence as part of the technology appraisal process. This institute is part of the UK NHS and its role is to provide patients, health professionals, and the public with authoritative, robust, and reliable guidance on current best practice. A monograph published in the Health Technology Assessment series provides further details on methods. ${ }^{4}$

We searched 15 electronic bibliographic databases from inception to October 2001. The bibliographies of relevant articles and submissions for sponsorship to the National Institute for Clinical Excellence were hand searched. Health services research resources were consulted through the internet. The search combined free text and thesaurus terms relating to central venous lines and ultrasonography. In smaller databases, searches were not restricted by publication type or study design. Filters used in Medline were aimed at identifying guidelines, systematic reviews, clinical trials, economic evaluations, and quality of life studies. Date and language restrictions were not applied. The full search strategy is available elsewhere. ${ }^{4}$

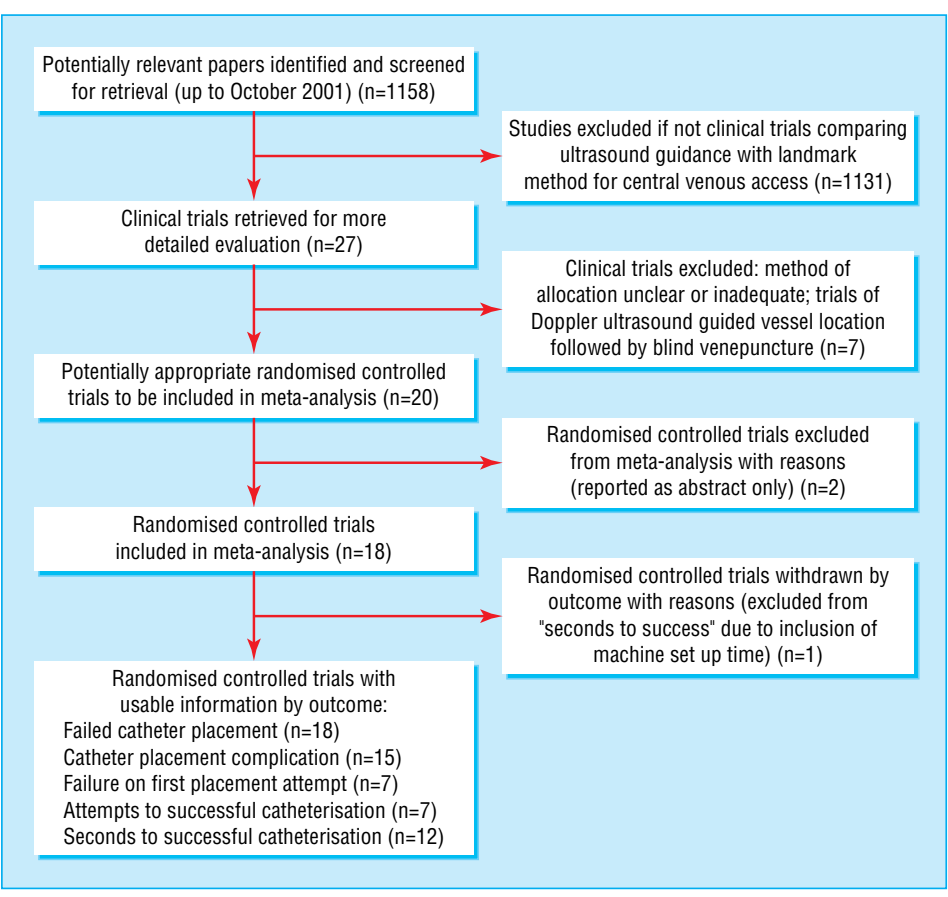

Fig 1 Study flow chart

\section{Selection and validity}

Inclusion criteria were: clinical effectiveness of two dimensional ultrasound guidance or Doppler ultrasound guidance for the placement of central venous lines; comparison of ultrasonography with the landmark method or the surgical cut-down procedure; inclusion of one or more of several outcomes-number of failed catheter placements, number of complications from catheter placement, risk of failure at first attempt, number of attempts to successful catheterisation, and time (seconds) to successful catheterisation. Only English language papers were selected, this being a rapid review to support decision making.

The abstracts of relevant citations were reviewed for potential randomised controlled trials. Trials were included unless the generation of allocation sequence was inadequate. ${ }^{5}$

Study quality was assessed by a component approach. ${ }^{6}$ When reported, allocation concealment and the method of generation of the allocation sequence were recorded, to assess the potential for selection bias. To assess the potential for attrition bias, we recorded whether an intention to treat analysis was performed.

\section{Data}

\section{Abstraction}

Data abstraction was based on reported summary statistics for the intention to treat population. Two coworkers extracted data independently, and discrepancies were resolved by consensus. The numbers of catheters and patients were abstracted as reported, as were data on mechanical complications. The numbers of patients with complications were pooled for meta-analysis. The numbers of catheter placements, rather than the numbers of patients, were pooled for analysis. Data for adults and children were pooled separately, as were alternative insertion sites.

\section{Analysis}

Treatment effect sizes and 95\% confidence intervals were calculated for each randomised comparison for each outcome. Relative risks were calculated for dichotomous outcomes, and weighted mean differences were calculated for continuous outcomes. Statistical heterogeneity was analysed to assess whether the observed variance in effect size between studies was greater than that expected by chance.

\section{Results}

We identified 27 trials. None reported allocation concealment. Three were excluded because the method of allocation was unclear and the trials were not described as randomised, and two were excluded because they had inadequate methods for generation of allocation sequence. Two prospective trials were rejected because vessels were located by Doppler ultrasound guidance followed by blind venepuncture. Two trials were rejected because they were reported in abstract form only. We therefore included 18 studies in our review (fig 1).

Table 1 lists participants' characteristics, interventions, operator experience, outcome measures, and quality of components for each trial. The trials 
Table 1 Participants, interventions, outcomes, and quality components of 18 randomised controlled trials of ultrasound guidance for central venous catheterisation

\begin{tabular}{|c|c|c|c|c|c|c|c|}
\hline Study & Setting & Participants & Comparison (entry site) & Outcomes measured & Operator experience & Randomisation method & $\begin{array}{c}\text { Exclusions after } \\
\text { randomisation }\end{array}$ \\
\hline $\begin{array}{l}\text { Alderson et } \\
\text { al1993 }\end{array}$ & $\begin{array}{l}\text { Canadian urban } \\
\text { children's } \\
\text { hospital }\end{array}$ & $\begin{array}{l}\text { Infants ( }<2 \text { years) } \\
\text { undergoing cardiac } \\
\text { surgery; disease and risk } \\
\text { not reported }\end{array}$ & $\begin{array}{l}\text { Two dimensional } \\
\text { ultrasound guidance } v \\
\text { landmark method } \\
\text { (internal jugular vein) }\end{array}$ & $\begin{array}{l}\text { Number of failed catheter } \\
\text { placements, number of } \\
\text { complications }\end{array}$ & $\begin{array}{l}\text { Experienced cardiac } \\
\text { anaesthetist }\end{array}$ & Not reported & None \\
\hline $\begin{array}{r}\text { Bold et al } \\
1998^{\mathrm{w} 2}\end{array}$ & $\begin{array}{l}\text { US tertiary care, } \\
\text { outpatient } \\
\text { oncology centre }\end{array}$ & $\begin{array}{l}\text { Adult chemotherapy } \\
\text { patients (cancer types } \\
\text { not reported); high risk } \\
\text { for failure or } \\
\text { complications }\end{array}$ & $\begin{array}{l}\text { Doppler ultrasound } \\
\text { guidance } v \text { landmark } \\
\text { method (subclavian vein) }\end{array}$ & $\begin{array}{l}\text { Number of failed catheter } \\
\text { placements }\end{array}$ & $\begin{array}{l}18 \text { surgical oncology } \\
\text { fellows (postgraduate } \\
\text { year } 6-10) \text {. Instruction in } \\
\text { use of smart needle and } \\
\text { "demonstrated } \\
\text { competence" in use of } \\
\text { Doppler probe }\end{array}$ & $\begin{array}{l}\text { Computer generated } \\
\text { block randomisation }\end{array}$ & None \\
\hline $\begin{array}{l}\text { Branger et al } \\
1994^{\mathrm{w} 3}\end{array}$ & $\begin{array}{l}\text { French teaching } \\
\text { hospital }\end{array}$ & $\begin{array}{l}\text { Patients needing central } \\
\text { venous catheterisation } \\
\text { for haemodialysis, } \\
\text { apheresis, or parenteral } \\
\text { nutrition (disease not } \\
\text { reported), low risk of } \\
\text { complications (high risk } \\
\text { patients excluded) }\end{array}$ & $\begin{array}{l}\text { Doppler ultrasound } \\
\text { guidance } v \text { landmark } \\
\text { method (internal jugular } \\
\text { vein and subclavian vein) }\end{array}$ & $\begin{array}{l}\text { Number of failed catheter } \\
\text { placements, number of } \\
\text { attempts to successful } \\
\text { catheterisation, time to } \\
\text { successful } \\
\text { catheterisation }\end{array}$ & $\begin{array}{l}14 \text { junior postgraduate } \\
\text { students with fewer than } \\
5 \text { years' clinical } \\
\text { experience, and } 8 \text { senior } \\
\text { staff with more than } 5 \\
\text { years' experience, from } \\
\text { nephrology, emergency, } \\
\text { and intensive care. } \\
\text { Taught the Doppler } \\
\text { technique over two } \\
\text { weeks, achieved at least } \\
\text { one venous } \\
\text { catheterisation before } \\
\text { entering study }\end{array}$ & Random tables & None \\
\hline $\begin{array}{l}\text { Gilbert et al } \\
1995^{\mathrm{w} 4}\end{array}$ & $\begin{array}{l}\text { US tertiary care, } \\
\text { teaching hospital }\end{array}$ & $\begin{array}{l}\text { Adult patients (disease } \\
\text { not reported) at high risk } \\
\text { from complications } \\
\text { (obesity or } \\
\text { coagulopathy) }\end{array}$ & $\begin{array}{l}\text { Doppler ultrasound } \\
\text { guidance } v \text { landmark } \\
\text { method (internal jugular } \\
\text { vein) }\end{array}$ & $\begin{array}{l}\text { Number of failed catheter } \\
\text { placements, number of } \\
\text { complications, failure on } \\
\text { first attempt, time to } \\
\text { successful } \\
\text { catheterisation }\end{array}$ & $\begin{array}{l}\text { Number not reported. } \\
\text { Junior housestaff } \\
\text { "relatively inexperienced } \\
\text { in using either } \\
\text { technique" }\end{array}$ & Not reported & None \\
\hline $\begin{array}{c}\text { Gratz et al } \\
1994^{\text {w5 }}\end{array}$ & $\begin{array}{l}\text { US tertiary care, } \\
\text { teaching hospital }\end{array}$ & $\begin{array}{l}\text { Patients for } \\
\text { cardiothoracic or } \\
\text { vascular surgery (age } \\
\text { and disease not } \\
\text { reported) }\end{array}$ & $\begin{array}{l}\text { Doppler ultrasound } \\
\text { guidance } v \text { landmark } \\
\text { method (internal jugular } \\
\text { vein) }\end{array}$ & $\begin{array}{l}\text { Number of failed catheter } \\
\text { placements, number of } \\
\text { complications, failure on } \\
\text { first attempt, number of } \\
\text { attempts to successful } \\
\text { catheterisation, time to } \\
\text { successful } \\
\text { catheterisation }\end{array}$ & $\begin{array}{l}\text { Number not reported; } \\
\text { "experienced } \\
\text { anesthesiologists" }\end{array}$ & Not reported & 1 of 41 \\
\hline $\begin{array}{l}\text { Gualtieri et al } \\
1995^{\mathrm{w} 6}\end{array}$ & $\begin{array}{l}\text { US urban } \\
\text { teaching hospital }\end{array}$ & $\begin{array}{l}\text { Critical care patients } \\
\text { undergoing } \\
\text { non-emergency } \\
\text { procedures (age, } \\
\text { disease, and risk not } \\
\text { reported) }\end{array}$ & $\begin{array}{l}\text { Two dimensional } \\
\text { ultrasound guidance } v \\
\text { landmark method } \\
\text { (subclavian vein) }\end{array}$ & $\begin{array}{l}\text { Number of failed catheter } \\
\text { placements; number of } \\
\text { complications }\end{array}$ & $\begin{array}{l}18 \text { physicians with }<30 \\
\text { procedures }\end{array}$ & Random number & 1 of 53 \\
\hline $\begin{array}{l}\text { Hilty et al } \\
1997^{\mathrm{w} 7}\end{array}$ & $\begin{array}{l}\text { US urban } \\
\text { teaching hospital }\end{array}$ & $\begin{array}{l}\text { Patients undergoing } \\
\text { cardiopulmonary } \\
\text { resuscitation (age, } \\
\text { disease, and risk not } \\
\text { reported) }\end{array}$ & $\begin{array}{l}\text { Two dimensional } \\
\text { ultrasound guidance } v \\
\text { landmark method } \\
\text { (femoral vein) }\end{array}$ & $\begin{array}{l}\text { Number of failed catheter } \\
\text { placements, failure on } \\
\text { first attempt, number of } \\
\text { attempts to successful } \\
\text { catheterisation, time to } \\
\text { successful } \\
\text { catheterisation }\end{array}$ & $\begin{array}{l}2 \text { emergency medicine } \\
\text { residents in postgraduate } \\
\text { years } 3 \text { and } 4.15-20 \\
\text { procedures using } \\
\text { landmark method; 6-10 } \\
\text { procedures using } \\
\text { ultrasonography }\end{array}$ & $\begin{array}{l}\text { Computer generated } \\
\text { randomisation chart }\end{array}$ & None \\
\hline $\begin{array}{l}\text { Lefrant et al } \\
1998^{\text {w8 }}\end{array}$ & $\begin{array}{l}\text { French teaching } \\
\text { hospital }\end{array}$ & $\begin{array}{l}\text { Critically ill adults } \\
\text { undergoing } \\
\text { non-emergency } \\
\text { procedures (disease and } \\
\text { risk not reported) }\end{array}$ & $\begin{array}{l}\text { Doppler ultrasound } \\
\text { guidance } v \text { landmark } \\
\text { method (subclavian vein) }\end{array}$ & $\begin{array}{l}\text { Number of failed catheter } \\
\text { placements, number of } \\
\text { complications, failure on } \\
\text { first attempt }\end{array}$ & $\begin{array}{l}1 \text { staff anaesthesiologist, } \\
\text { untrained in Doppler } \\
\text { guidance before study }\end{array}$ & Random number & None \\
\hline $\begin{array}{l}\text { Mallory et al } \\
1990^{\mathrm{w} 9}\end{array}$ & $\begin{array}{l}\text { US tertiary care, } \\
\text { teaching hospital }\end{array}$ & $\begin{array}{l}\text { Critically ill adult patients } \\
\text { in intensive care; high } \\
\text { and low risk (disease not } \\
\text { reported) }\end{array}$ & $\begin{array}{l}\text { Two dimensional } \\
\text { ultrasound guidance } v \\
\text { landmark method } \\
\text { (internal jugular vein) }\end{array}$ & $\begin{array}{l}\text { Number of failed catheter } \\
\text { placements, failure on } \\
\text { first attempt }\end{array}$ & $\begin{array}{l}\text { Senior intensive care unit } \\
\text { staff and critical care } \\
\text { fellows. Number not } \\
\text { reported. Mean } 6 \text { years' } \\
\text { experience }\end{array}$ & Not reported & None \\
\hline $\begin{array}{l}\text { Nadig et al } \\
1998^{\text {w10 }}\end{array}$ & $\begin{array}{l}\text { German teaching } \\
\text { hospital }\end{array}$ & $\begin{array}{l}\text { Dialysis patients (age, } \\
\text { disease, and risk level } \\
\text { not reported) }\end{array}$ & $\begin{array}{l}\text { Two dimensional } \\
\text { ultrasound guidance } v \\
\text { two dimensional } \\
\text { ultrasound guidance for } \\
\text { vessel location followed } \\
\text { by blind venepuncture } \\
\text { (internal jugular vein) }\end{array}$ & $\begin{array}{l}\text { Number of failed catheter } \\
\text { placements, number of } \\
\text { complications, failure on } \\
\text { first attempt, time to } \\
\text { successful } \\
\text { catheterisation }\end{array}$ & $\begin{array}{l}\text { Physicians; clinical } \\
\text { experience 1-7 years }\end{array}$ & By lot & None \\
\hline $\begin{array}{l}\text { Slama et al } \\
1997^{\text {w11 }}\end{array}$ & $\begin{array}{l}\text { French } \\
\text { university } \\
\text { hospital }\end{array}$ & $\begin{array}{l}\text { Adults in intensive care } \\
\text { requiring cannulation of } \\
\text { internal jugular vein } \\
\text { (disease and risk } \\
\text { assessment not reported) }\end{array}$ & $\begin{array}{l}\text { Two dimensional } \\
\text { ultrasound guidance } v \\
\text { landmark method } \\
\text { (internal jugular vein) }\end{array}$ & $\begin{array}{l}\text { Number of failed catheter } \\
\text { placements, number of } \\
\text { complications, failure on } \\
\text { first attempt; time to } \\
\text { successful } \\
\text { catheterisation }\end{array}$ & $\begin{array}{l}\text { Junior house staff } \\
\text { (interns or residents) } \\
\text { under the direct } \\
\text { supervision of senior } \\
\text { physician after at least } \\
\text { three demonstrations by } \\
\text { experienced operator and } \\
\text { three attempts of right } \\
\text { internal jugular vein } \\
\text { using landmark method }\end{array}$ & Not reported & None \\
\hline
\end{tabular}


Table 1 Participants, interventions, outcomes, and quality components of 18 randomised controlled trials of ultrasound guidance for central venous catheterisation-continued from previous page

\begin{tabular}{|c|c|c|c|c|c|c|c|}
\hline Study & Setting & Participants & Comparison (entry site) & Outcomes measured & Operator experience & Randomisation method & $\begin{array}{c}\text { Exclusions after } \\
\text { randomisation }\end{array}$ \\
\hline $\begin{array}{l}\text { Soyer et al } \\
1993^{\text {w12 }}\end{array}$ & French hospital & $\begin{array}{l}\text { Adult patients with liver } \\
\text { dysfunction requiring } \\
\text { transjugular liver biopsy } \\
\text { (risk assessment not } \\
\text { reported) }\end{array}$ & $\begin{array}{l}\text { Two dimensional } \\
\text { ultrasound guidance } v \\
\text { landmark method } \\
\text { (internal jugular vein) }\end{array}$ & $\begin{array}{l}\text { Number of failed catheter } \\
\text { placements, number of } \\
\text { complications, number of } \\
\text { attempts to successful } \\
\text { catheterisation, time to } \\
\text { successful } \\
\text { catheterisation }\end{array}$ & $\begin{array}{l}2 \text { radiologists with same } \\
\text { experience (not } \\
\text { quantified) }\end{array}$ & Not reported & None \\
\hline $\begin{array}{l}\text { Sulek et al } \\
2000^{\mathrm{w} 13}\end{array}$ & $\begin{array}{l}\text { US university } \\
\text { affiliated } \\
\text { hospital; } \\
\text { operating room }\end{array}$ & $\begin{array}{l}\text { Adult patients scheduled } \\
\text { for elective abdominal, } \\
\text { vascular, or } \\
\text { cardiothoracic } \\
\text { procedures with general } \\
\text { anaesthesia and } \\
\text { mechanical ventilation in } \\
\text { whom central venous } \\
\text { cannulation was clinically } \\
\text { indicated (disease and } \\
\text { risk assessment not } \\
\text { reported) }\end{array}$ & $\begin{array}{l}\text { Two dimensional } \\
\text { ultrasound guidance } v \\
\text { landmark method } \\
\text { (internal jugular vein) }\end{array}$ & $\begin{array}{l}\text { Number of failed catheter } \\
\text { placements, number of } \\
\text { complications, number of } \\
\text { attempts to successful } \\
\text { catheterisation, time to } \\
\text { successful } \\
\text { catheterisation }\end{array}$ & $\begin{array}{l}\text { Anaesthetist. All } \\
\text { operators experienced in } \\
\text { cannulation of internal } \\
\text { jugular vein (at least } 60 \\
\text { catheter placements) } \\
\text { with known expertise in } \\
\text { use of ultrasound guided } \\
\text { internal jugular vein } \\
\text { technique }\end{array}$ & $\begin{array}{l}\text { Computer generated } \\
\text { randomisation table }\end{array}$ & None \\
\hline $\begin{array}{l}\text { Teichgräber et al } \\
1997^{\text {w14 }}\end{array}$ & $\begin{array}{l}\text { German } \\
\text { university } \\
\text { teaching hospital }\end{array}$ & $\begin{array}{l}\text { Patients undergoing } \\
\text { routine catheterisation of } \\
\text { internal jugular vein } \\
\text { (age, disease, and } \\
\text { risk-assessment not } \\
\text { reported) }\end{array}$ & $\begin{array}{l}\text { Two dimensional } \\
\text { ultrasound guidance } v \\
\text { landmark method } \\
\text { (internal jugular vein) }\end{array}$ & $\begin{array}{l}\text { Number of failed catheter } \\
\text { placements, number of } \\
\text { complications }\end{array}$ & $\begin{array}{l}\text { Physicians. Number and } \\
\text { experience not reported }\end{array}$ & Not reported & None \\
\hline $\begin{array}{l}\text { Troianos et al } \\
1991^{\text {w15 }}\end{array}$ & $\begin{array}{l}\text { US tertiary care, } \\
\text { teaching hospital }\end{array}$ & $\begin{array}{l}\text { Cardiothoracic surgical } \\
\text { patients (age, disease, } \\
\text { and risk factor not } \\
\text { reported) }\end{array}$ & $\begin{array}{l}\text { Two dimensional } \\
\text { ultrasound guidance } v \\
\text { landmark method } \\
\text { (internal jugular vein) }\end{array}$ & $\begin{array}{l}\text { Number of failed catheter } \\
\text { placements, number of } \\
\text { complications, failure on } \\
\text { first attempt, number of } \\
\text { attempts to successful } \\
\text { catheterisation, time to } \\
\text { successful } \\
\text { catheterisation }\end{array}$ & Not reported & Not reported & None \\
\hline $\begin{array}{l}\text { Verghese et al } \\
1999^{\text {w16 }}\end{array}$ & $\begin{array}{l}\text { US university } \\
\text { teaching hospital }\end{array}$ & $\begin{array}{l}\text { Infants scheduled for } \\
\text { cardiovascular surgery, } \\
<12 \text { months, weight } \\
<10 \mathrm{~kg} \text { (disease and risk } \\
\text { assessment not reported) }\end{array}$ & $\begin{array}{l}\text { Two dimensional } \\
\text { ultrasound guidance } v \\
\text { landmark method } \\
\text { (internal jugular vein) }\end{array}$ & $\begin{array}{l}\text { Number of failed catheter } \\
\text { placements, number of } \\
\text { complications, number of } \\
\text { attempts to successful } \\
\text { catheterisation, time to } \\
\text { successful } \\
\text { catheterisation }\end{array}$ & $\begin{array}{l}\text { Number not reported. } \\
\text { Board eligible } \\
\text { anaesthesia fellows who } \\
\text { had completed residency } \\
\text { training in anaesthesia }\end{array}$ & $\begin{array}{l}\text { Computer generated } \\
\text { randomisation table }\end{array}$ & None \\
\hline $\begin{array}{l}\text { Verghese et al } \\
2000^{\mathrm{w} 17}\end{array}$ & $\begin{array}{l}\text { US university } \\
\text { teaching hospital }\end{array}$ & $\begin{array}{l}45 \text { infants scheduled to } \\
\text { undergo internal jugular } \\
\text { cannulation during } \\
\text { cardiac surgery (disease } \\
\text { and risk assessment not } \\
\text { reported) }\end{array}$ & $\begin{array}{l}\text { Two dimensional } \\
\text { ultrasound guidance } v \\
\text { Doppler ultrasound } \\
\text { guidance } v \text { landmark } \\
\text { method (internal jugular } \\
\text { vein) }\end{array}$ & $\begin{array}{l}\text { Number of failed catheter } \\
\text { placements, number of } \\
\text { complications, time to } \\
\text { successful } \\
\text { catheterisation }\end{array}$ & $\begin{array}{l}\text { Number not reported. } \\
\text { Fellows in paediatric } \\
\text { anaesthesia }\end{array}$ & $\begin{array}{l}\text { Computer generated } \\
\text { randomisation table }\end{array}$ & None \\
\hline $\begin{array}{l}\text { Vucevic et al } \\
1994^{\mathrm{w} 18}\end{array}$ & British hospital & $\begin{array}{l}\text { Cardiac surgery and } \\
\text { intensive care unit } \\
\text { patients (age, disease, } \\
\text { and risk-assessment not } \\
\text { reported) }\end{array}$ & $\begin{array}{l}\text { Doppler ultrasound } \\
\text { guidance } v \text { landmark } \\
\text { method (internal jugular } \\
\text { vein) }\end{array}$ & $\begin{array}{l}\text { Number of failed catheter } \\
\text { placements, number of } \\
\text { complications, time to } \\
\text { successful } \\
\text { catheterisation }\end{array}$ & $\begin{array}{l}2 \text { consultant } \\
\text { anaesthetists; } 10 \\
\text { procedures }\end{array}$ & Not reported & None \\
\hline
\end{tabular}

included a total of 1646 people scheduled for central venous catheterisation. Ten studies investigated two dimensional ultrasound guidance compared with the landmark method and six investigated Doppler ultrasound guidance compared with the landmark method. One trial investigated two dimensional ultrasound guidance compared with blind venepuncture preceded by ultrasound guidance. One trial, with three arms, investigated two dimensional ultrasound guidance compared with Doppler ultrasound guidance and the landmark method. No studies compared two dimensional ultrasound guidance as a single procedure against surgical cut-down. Nine trials described adequate methods for generation of allocation sequence within the randomisation process. Two trials did not indicate an intention to treat analysis.

\section{Quantitative data synthesis}

Table 2 summarises the pooled results from the metaanalyses comparing two dimensional ultrasonography with the landmark method for both adults and infants in all five outcomes. Figure 2 shows graphical data for the primary outcome. Two dimensional ultrasound guidance was more effective for all five outcomes for internal jugular vein procedures in adults (relative risk reductions: $86 \%$ for failed catheter placements, $57 \%$ for complications with catheter placement, and $41 \%$ for failure on first attempt; all $\mathrm{P}<0.05$. Fewer attempts were required to successfully cannulate patients and significantly less time was needed. Limited evidence suggested two dimensional ultrasound guidance reduced the relative risk of failed catheter placements by $86 \%$ in the subclavian vein and $71 \%$ in the femoral vein. Three studies of this comparison for procedures on internal jugular veins in infants had relatively small sample sizes but suggested that ultrasonography was significantly more effective (relative risk reductions: $85 \%$ for failed catheter placements and $73 \%$ for complications with catheter placement).

Table 3 summarises the results of the meta-analyses comparing Doppler ultrasound guidance with the landmark method. For internal jugular vein procedures, Doppler ultrasound guidance significantly improved the chance of successful cannulation overall 
and on the first attempt. However, for cannulation of the subclavian vein, results significantly favoured the landmark method for relative risk of failed catheter placements and the mean number of seconds to successful catheterisation. Only one study of this comparison in infants was found (for internal jugular vein procedures), and this was too small to achieve statistical significance. No studies of this comparison in femoral vein procedures were identified for adults or infants.

In the absence of studies comparing two dimensional ultrasonography with Doppler ultrasonography in adults, we made an indirect comparison of the two estimated relative risks (table 4). ${ }^{7}$ The ratio of relative risks for the primary outcome, failed catheter placements, was 0.36 (0.11 to 1.19$)$ in favour of two dimensional ultrasonography for internal jugular vein procedures and 0.09 (0.02 to 0.38 ) for subclavian vein procedures.

\section{Discussion}

Our systematic review shows a clear benefit from two dimensional ultrasound guidance for central venous access compared with the landmark method. This is manifest in a lower technical failure rate (overall and on first attempt), a reduction in complications, and faster access. One explanation for these benefits is that ultrasonography clarifies the relative position of the needle, the vein, and its surrounding structures. The image offered by two dimensional ultrasonography allows the user to predict variant anatomy and to assess the patency of a target vein. The clinical effect of using ultrasound guidance was more significant when the internal jugular vein rather than the subclavian or femoral veins was cannulated, for which evidence was sparse. These results are similar to a previously published meta-analysis: however, that study inappropriately pooled the results from trials of both Doppler ultrasound guidance and two dimensional ultrasound guidance. $^{8}$ The evidence presented here favours the use of two dimensional ultrasound guidance for cannulation of the subclavian vein, with Doppler ultrasound guidance less successful and more time consuming than even the landmark method. It also proved more successful than Doppler ultrasound guidance or the landmark method when the internal jugular vein of infants was cannulated, the image aiding the navigation of diminutive anatomy; although this evidence came from only one study.

Ultrasound guidance is therefore likely to have benefits to patients with a reduction in the risks of the procedure, and they are less likely to undergo a prolonged, sometimes uncomfortable and possibly fruitless attempt at central venous cannulation. Potential benefits to NHS trusts are improvements in efficiency and reductions in costs of dealing with complications. To be weighed against this are the implications of advocating ultrasound guidance for central venous cannulation, such as a potential for deskilling in the landmark method that may be required in some emergency situations. Guidance from the National Institute for Clinical Excellence in this area states that it is important that "operators maintain their ability to use the landmark method and that the method continues to be taught alongside the 2-D ultrasound guided technique." ${ }^{.9}$ Financial and logistical

Table 2 Summary of significance of outcome measures for two dimensional (2-D) ultrasound guidance compared with landmark method for catheterisation

\begin{tabular}{|c|c|c|c|c|c|c|c|c|c|c|c|c|}
\hline \multirow[b]{3}{*}{ Variable } & \multicolumn{4}{|c|}{ Internal jugular vein } & \multicolumn{4}{|c|}{ Subclavian vein } & \multicolumn{4}{|c|}{ Femoral vein } \\
\hline & \multicolumn{2}{|c|}{ No of placements } & \multirow[b]{2}{*}{$\begin{array}{l}\text { Effect size } \\
(95 \% \mathrm{Cl})\end{array}$} & \multirow[b]{2}{*}{$P$ value } & \multicolumn{2}{|c|}{ No of placements } & \multirow[b]{2}{*}{$\begin{array}{l}\text { Effect size } \\
(95 \% \mathrm{CI})\end{array}$} & \multirow[b]{2}{*}{$\begin{array}{c}P \\
\text { value }\end{array}$} & \multicolumn{2}{|c|}{ No of placements } & \multirow[b]{2}{*}{$\begin{array}{l}\text { Effect size } \\
(95 \% \mathrm{Cl})\end{array}$} & \multirow[b]{2}{*}{$\begin{array}{c}P \\
\text { value }\end{array}$} \\
\hline & $\begin{array}{c}\text { 2-D } \\
\text { ultrasound } \\
\text { guidance }\end{array}$ & $\begin{array}{l}\text { Landmark } \\
\text { method }\end{array}$ & & & $\begin{array}{c}\text { 2-D } \\
\text { ultrasound } \\
\text { guidance }\end{array}$ & $\begin{array}{l}\text { Landmark } \\
\text { method }\end{array}$ & & & $\begin{array}{c}\text { Doppler } \\
\text { ultrasound } \\
\text { guidance }\end{array}$ & $\begin{array}{l}\text { Landmark } \\
\text { method }\end{array}$ & & \\
\hline \multicolumn{13}{|l|}{ Adults } \\
\hline \multicolumn{13}{|l|}{ Relative risk: } \\
\hline $\begin{array}{l}\text { Failed catheter } \\
\text { placement }\end{array}$ & 296 & 312 & $0.14(0.06$ to 0.33$)$ & $<0.0001$ & 25 & 27 & 0.14 (0.04 to 0.57$)$ & 0.006 & 20 & 20 & 0.29 (0.07 to 1.21$)$ & 0.09 \\
\hline $\begin{array}{l}\text { Complication with } \\
\text { placement }\end{array}$ & 284 & 295 & $0.43(0.22$ to 0.87$)$ & 0.02 & 25 & 27 & 0.10 (0.01 to 0.71$)$ & 0.02 & - & - & NA & NA \\
\hline $\begin{array}{l}\text { Failure on first } \\
\text { attempt }\end{array}$ & 162 & 179 & $0.59(0.39$ to 0.88$)$ & 0.009 & - & - & NA & NA & - & - & NA & NA \\
\hline \multicolumn{13}{|l|}{ Mean No: } \\
\hline $\begin{array}{l}\text { Attempts to } \\
\text { successful } \\
\text { catheterisation }\end{array}$ & 131 & 136 & $\begin{array}{c}-1.50 \\
(-2.53 \text { to }-0.47)\end{array}$ & 0.004 & - & - & NA & NA & 20 & 20 & $\begin{array}{c}-2.70 \\
(-5.26 \text { to }-0.14)\end{array}$ & 0.04 \\
\hline $\begin{array}{l}\text { Seconds to } \\
\text { successful } \\
\text { catheterisation }\end{array}$ & 180 & 192 & $\begin{array}{c}-69.33 \\
(-92.36 \text { to }-46.31)\end{array}$ & $<0.0001$ & - & - & NA & NA & 20 & 20 & $\begin{array}{c}-3.20 \\
(-43.27 \text { to } 36.87)\end{array}$ & 0.9 \\
\hline \multicolumn{13}{|l|}{ Infants } \\
\hline \multicolumn{13}{|l|}{ Relative risk: } \\
\hline Failed placement & 79 & 88 & $0.15(0.03$ to 0.64$)$ & 0.01 & - & - & NA & NA & - & - & NA & NA \\
\hline $\begin{array}{l}\text { Complication with } \\
\text { placement }\end{array}$ & 79 & 88 & 0.27 (0.08 to 0.91$)$ & 0.03 & - & - & NA & NA & - & - & NA & NA \\
\hline $\begin{array}{l}\text { Failure on first } \\
\text { attempt }\end{array}$ & - & - & NA & NA & - & - & NA & NA & - & - & NA & $\mathrm{NA}$ \\
\hline \multicolumn{13}{|l|}{ Mean No: } \\
\hline $\begin{array}{l}\text { Attempts to } \\
\text { successful } \\
\text { catheterisation }\end{array}$ & 43 & 52 & $\begin{array}{c}-2.00 \\
(-2.78 \text { to }-1.22)\end{array}$ & $<0.0001$ & - & - & NA & NA & - & - & NA & NA \\
\hline $\begin{array}{l}\text { Seconds to } \\
\text { successful } \\
\text { catheterisation }\end{array}$ & 59 & 68 & $\begin{array}{c}-349.38 \\
(-801.89 \text { to } 103.13)\end{array}$ & 0.13 & - & - & NA & NA & - & - & NA & NA \\
\hline
\end{tabular}

$\mathrm{NA}=$ not available. All outcomes favoured ultrasound guidance (relative risk $<1$ ). 
Table 3 Summary of significance of outcome measures for Doppler ultrasound guidance compared with landmark method for catheterisation

\begin{tabular}{|c|c|c|c|c|c|c|c|c|c|c|c|c|}
\hline \multirow[b]{3}{*}{ Variable } & \multicolumn{4}{|c|}{ Internal jugular vein } & \multicolumn{4}{|c|}{ Subclavian vein } & \multicolumn{4}{|c|}{ Femoral vein } \\
\hline & \multicolumn{2}{|c|}{ No of placements } & \multirow[b]{2}{*}{$\begin{array}{c}\text { Effect size } \\
(95 \% \mathrm{CI})\end{array}$} & \multirow[b]{2}{*}{$P$ value } & \multicolumn{2}{|c|}{ No of placements } & \multirow[b]{2}{*}{$\begin{array}{l}\text { Effect size } \\
(95 \% \mathrm{Cl})\end{array}$} & \multirow[b]{2}{*}{$\begin{array}{c}P \\
\text { value }\end{array}$} & \multicolumn{2}{|c|}{ No of placements } & \multirow[b]{2}{*}{$\begin{array}{l}\text { Effect } \\
\text { size } \\
(95 \% \mathrm{Cl})\end{array}$} & \multirow[b]{2}{*}{$\begin{array}{c}P \\
\text { value }\end{array}$} \\
\hline & $\begin{array}{l}\text { Doppler } \\
\text { ultrasound } \\
\text { guidance }\end{array}$ & $\begin{array}{c}\text { Landmark } \\
\text { method }\end{array}$ & & & $\begin{array}{l}\text { Doppler } \\
\text { ultrasound } \\
\text { guidance }\end{array}$ & $\begin{array}{l}\text { Landmark } \\
\text { method }\end{array}$ & & & $\begin{array}{c}\text { Doppler } \\
\text { ultrasound } \\
\text { guidance }\end{array}$ & $\begin{array}{l}\text { Landmark } \\
\text { method }\end{array}$ & & \\
\hline \multicolumn{13}{|l|}{ Adults } \\
\hline \multicolumn{13}{|l|}{ Relative risk: } \\
\hline $\begin{array}{l}\text { Failed catheter } \\
\text { placement }\end{array}$ & 86 & 99 & 0.39 (0.17 to 0.92$)$ & 0.03 & 310 & 314 & $1.48(1.03 \text { to } 2.14)^{\star}$ & $0.03^{*}$ & - & - & NA & NA \\
\hline $\begin{array}{l}\text { Complication with } \\
\text { placement }\end{array}$ & 89 & 89 & 0.43 (0.17 to 1.05 ) & 0.06 & 262 & 264 & 0.57 (0.11 to 2.88$)$ & 0.5 & - & - & NA & NA \\
\hline $\begin{array}{l}\text { Failure on first } \\
\text { attempt }\end{array}$ & 52 & 64 & 0.57 (0.37 to 0.88 ) & 0.01 & 143 & 143 & $1.04(0.76 \text { to } 1.43)^{*}$ & $0.8^{*}$ & - & - & NA & NA \\
\hline \multicolumn{13}{|l|}{ Mean No: } \\
\hline $\begin{array}{l}\text { Attempts to } \\
\text { successful } \\
\text { catheterisation }\end{array}$ & 34 & 35 & $\begin{array}{c}-0.59 \\
(-1.82 \text { to } 0.65)\end{array}$ & 0.4 & 48 & 50 & $\begin{array}{c}-0.4 \\
(-0.61 \text { to }-0.19)\end{array}$ & 0.0002 & - & - & NA & NA \\
\hline $\begin{array}{l}\text { Seconds to } \\
\text { successful } \\
\text { catheterisation }\end{array}$ & 86 & 99 & $\begin{array}{c}34.86 \\
(-54.49 \text { to } 124.21)^{\star}\end{array}$ & $0.4^{*}$ & 48 & 50 & $\begin{array}{c}209.00 \\
(175.48 \text { to } 242.52)^{\star}\end{array}$ & $<0.0001^{*}$ & - & - & NA & NA \\
\hline \multicolumn{13}{|l|}{ Infants } \\
\hline $\begin{array}{l}\text { No of failed catheter } \\
\text { placements }\end{array}$ & 13 & 16 & $1.23(0.30 \text { to } 5.11)^{*}$ & $0.8^{*}$ & - & - & NA & NA & - & - & NA & NA \\
\hline $\begin{array}{l}\text { No of complications } \\
\text { from placement }\end{array}$ & 13 & 16 & 0.82 (0.16 to 4.20$)$ & 0.8 & - & - & NA & NA & - & - & NA & NA \\
\hline $\begin{array}{l}\text { Risk of failure on first } \\
\text { attempt }\end{array}$ & - & - & NA & NA & - & - & NA & NA & - & - & NA & NA \\
\hline $\begin{array}{l}\text { Mean No of attempts } \\
\text { to successful } \\
\text { catheterisation }\end{array}$ & - & - & NA & $N A$ & - & - & NA & NA & - & - & $N A$ & NA \\
\hline $\begin{array}{l}\text { Mean No of seconds to } \\
\text { successful } \\
\text { catheterisation }\end{array}$ & 13 & 16 & $\begin{array}{c}138.00 \\
(-114.72 \text { to } 390.72)^{*}\end{array}$ & $0.3^{*}$ & - & - & NA & NA & - & - & NA & NA \\
\hline
\end{tabular}

NA=not available. Relative risks $<1$ favour Doppler ultrasound guidance.

*Outcome favours landmark method.

implications for the NHS are provision of sufficient ultrasound machines and staff training.

Economic modelling, undertaken for the Health Technology Assessment programme, indicated that using ultrasound guidance for venepuncture in central venous access was likely to save $£ 2000$ (\$3249; €2840) of NHS resources for every 1000 procedures. ${ }^{4}$ The model incorporated the inevitable costs of purchasing machines and training staff. The net resource saving was attributable to savings from the need to treat fewer complications and notional savings from less time spent by clinicians and nurses achieving successful cannulation and dealing with complications, with all the implications for reduced use of expensive time in theatres and intensive care units. Although wider use of two dimensional ultrasound guidance for central venous access is unlikely to achieve hard cash savings for the NHS, the opportunity cost savings are genuine and relevant.

Table 4 Summary of ratio of relative risks for two dimensional ultrasound guidance indirectly compared with Doppler ultrasound guidance

\begin{tabular}{|c|c|c|c|c|c|c|}
\hline \multirow[b]{2}{*}{ Variable } & \multicolumn{2}{|c|}{ Internal jugular vein } & \multicolumn{2}{|c|}{ Subclavian vein } & \multicolumn{2}{|c|}{ Femoral vein } \\
\hline & Effect size $(95 \% \mathrm{CI})$ & P value & Effect size $(95 \% \mathrm{CI})$ & $P$ value & Effect size $(95 \% \mathrm{CI})$ & $P$ value \\
\hline \multicolumn{7}{|l|}{ Adults } \\
\hline \multicolumn{7}{|l|}{ Relative risk: } \\
\hline Failed catheter placement & 0.36 (0.11 to 1.19$)$ & 0.09 & $0.09(0.02$ to 0.38$)$ & 0.0008 & NA & NA \\
\hline Complication with placement & $1.00(0.32$ to 3.13$)$ & 1.00 & 0.18 (0.01 to 2.57$)$ & 0.2 & NA & NA \\
\hline Failure on first attempt & 1.04 (0.57 to 1.88$)$ & 0.9 & NA & NA & NA & NA \\
\hline \multicolumn{7}{|l|}{ Mean No: } \\
\hline $\begin{array}{l}\text { Attempts to successful } \\
\text { catheterisation }\end{array}$ & $-0.91(-2.52$ to 0.70$)$ & 0.3 & NA & NA & NA & NA \\
\hline $\begin{array}{l}\text { Seconds to successful } \\
\text { catheterisation }\end{array}$ & $-104(-196$ to -12$)$ & 0.03 & NA & NA & NA & NA \\
\hline \multicolumn{7}{|l|}{ Infants } \\
\hline \multicolumn{7}{|l|}{ Relative risk: } \\
\hline Failed catheter placement & $0.12(0.02$ to 0.98$)$ & 0.048 & NA & NA & NA & NA \\
\hline Complication from placement & 0.33 (0.04 to 2.52) & 0.3 & NA & NA & NA & NA \\
\hline Failure on first attempt & NA & NA & NA & NA & NA & NA \\
\hline \multicolumn{7}{|l|}{ Mean (No): } \\
\hline $\begin{array}{l}\text { Attempts to successful } \\
\text { catheterisation }\end{array}$ & NA & NA & NA & NA & NA & NA \\
\hline $\begin{array}{l}\text { Seconds to successful } \\
\text { catheterisation }\end{array}$ & $-487.38(-1006.00$ to 31.00$)$ & 0.06 & NA & NA & NA & NA \\
\hline
\end{tabular}




\section{What is already known on this topic}

Hundreds of thousands of central venous lines are placed in patients every year in NHS hospitals

Complication and failure rates vary, and deaths have been reported

\section{What this study adds}

Catheterisation under two dimensional ultrasound guidance is quicker and safer than the landmark method in both adults and children

Two dimensional ultrasound guidance is more effective than Doppler ultrasound guidance for more difficult procedures

Contributors: DH and NC designed the review, screened search results, screened retrieved papers against inclusion criteria, appraised quality of papers, abstracted data from papers, analysed data, provided a methodological perspective in the interpretation of data, and wrote the review. RMcW and $\mathrm{AD}$ obtained and screened data on unpublished studies, provided a clinical perspective in the interpretation of data, provided general advice on the review, and wrote the review. SP coordinated the review, provided general advice on the review, and secured funding. $\mathrm{CB}$ designed the review, developed the search strategy, undertook searches, organised retrieval of papers, and wrote the review. ST provided a clinical perspective in the interpretation of data, provided general advice on the review, and wrote the review. KB, nurse consultant in critical care medicine (Sheffield Teaching Hospitals NHS Trust), provided a clinical perspective in the interpretation of data and provided general advice on the review.

Funding: The UK National Coordinating Centre for Health Technology Assessment programme funded the study.

Competing interests: $\mathrm{RMcW}$ has received honorariums from Sonosite for lecturing at training days.

1 Elliot TSJ, Faroqui MH, Armstrong RF, Hanson GC. Guidelines for good practice in central venous catheterization.J Hosp Infect 1994;28:163-76.

2 Callum KG, Whimster F. Interventional vascular radiology and interventional neurovascular radiology: a report of the National Confidential Enquiry into Perioperative Deaths. Data collection period 1 Apr 1998 to 31 Mar 1999. London, NCEPOD, 2000.

3 Trottier SJ, Veremakis C, O'Brien J, Auer AI. Femoral deep vein thrombosis associated with central venous catheterization: results from a thrombosis associated with central venous catheterization:

4 Calvert N, Hind D, McWilliams RG, Thomas SM, Beverley C, Davidson A. The effectiveness and cost-effectiveness of ultrasound locating devices for central venous access: a systematic review. Health Technol Asses 2003:7(12).

5 Schulz KF, Chalmers I, Hayes RJ, Altman DG. Empirical evidence of bias dimensions of methodological quality associated with estimates of treatment effects in controlled trials. JAMA 2003;273:408-12.

6 Khan KS, ter Riet G, Glanville J, Sowden AJ, Kleijnen J. Undertaking systematic reviews of research on effectiveness: CRD's ouidance for those carrying out or commissioning reviecus. York. NHS Centre for Reviews and Dissemnation, University of York, 2001.

\begin{tabular}{|c|c|c|c|c|c|}
\hline $\begin{array}{r}\text { Two } \\
\text { ultraso }\end{array}$ & $\begin{array}{l}\text { dimensional } \\
\text { nography }(\mathrm{n} / \mathrm{N})\end{array}$ & $\begin{array}{l}\text { Landmark } \\
\text { method }(n / N)\end{array}$ & $\begin{array}{c}\text { Relative risk } \\
\text { (95\% Cl random) }\end{array}$ & $\begin{array}{l}\text { Weight } \\
(\%)\end{array}$ & $\begin{array}{c}\text { Relative risk } \\
(95 \% \mathrm{Cl} \text { random) }\end{array}$ \\
\hline \multicolumn{6}{|c|}{ Failed catheter placement (adults, internal jugular vein) } \\
\hline Mallory et al $1990^{\text {w9 }}$ & $0 / 12$ & $6 / 17$ & & 8.8 & $0.11(0.01$ to 1.73$)$ \\
\hline Nadig et al $1998^{\text {w10 }}$ & $0 / 36$ & $13 / 37$ & & 8.8 & $0.04(0.00$ to 0.62$)$ \\
\hline Slama et al $1997^{\mathrm{w} 11}$ & $0 / 37$ & $10 / 42$ & & 8.7 & $0.05(0.00$ to 0.89$)$ \\
\hline Soyer et al $1993^{w 12}$ & $0 / 24$ & $5 / 23$ & & 8.5 & $0.09(0.01$ to 1.49$)$ \\
\hline Sulek et al $2000^{\text {w13 }}$ & $3 / 60$ & $5 / 60$ & $\longrightarrow$ & 28.6 & $0.60(0.15$ to 2.40$)$ \\
\hline Teichgräber et al $1997^{\mathrm{w} 14}$ & ${ }^{4} 2 / 50$ & $26 / 50$ & - & 28.7 & $0.08(0.02$ to 0.31$)$ \\
\hline Troianos et al $1991^{\mathrm{w} 15}$ & $0 / 77$ & $3 / 83$ & - & 7.9 & $0.15(0.01$ to 2.93$)$ \\
\hline Total (95\% Cl) & $5 / 296$ & $68 / 312$ & - & 100.0 & $0.14(0.06$ to 0.33$)$ \\
\hline
\end{tabular}

Test for overall effect: $z=-4.50, P=0.0001$

\begin{tabular}{|c|c|c|c|}
\hline \multicolumn{4}{|c|}{ Failed catheter placement (adults, subclavian vein) } \\
\hline Gualtieri et al $1995^{w 6}$ & $2 / 25$ & $15 / 27$ & $100.00 .14(0.04$ to 0.57$)$ \\
\hline Total $(95 \% \mathrm{Cl})$ & $2 / 25$ & $15 / 27$ & $100.00 .14(0.04$ to 0.57$)$ \\
\hline
\end{tabular}

Test for heterogeneity: $\chi^{2}=0.0, \mathrm{df}=0$

Test for overall effect: $z=-2.77, P=0.006$

Failed catheter placement (adults, femoral vein)

Hilty et al $1997^{\mathrm{w} 7} \quad 2 / 20 \quad 7 / 20$

Total $(95 \% \mathrm{Cl}) \quad 2 / 20$

Test for heterogeneity: $\chi^{2}=0.0, \mathrm{df}=0$

$7 / 20$

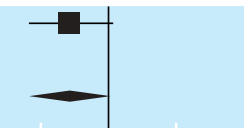

$100.0 \quad 0.29(0.07$ to 1.21$)$

$100.00 .29(0.07$ to 1.21$)$

Test for overall effect: $z=-1.70, P=0.09$

Failed catheter placement (infants, internal jugular vein)

Alderson et al $1993^{\mathrm{w} 1} \quad$ 0/20

Verghese et al 1999w16 $\quad 0 / 43$

Verghese et al 2000 ${ }^{\text {w17 }} \quad 1 / 16$

Total $(95 \% \mathrm{Cl}) \quad 1 / 79$

Test for heterogeneity: $\chi^{2}=1.36, d f=2, P=0.51$

Test for overall effect: $z=-2.56, P=0.01$

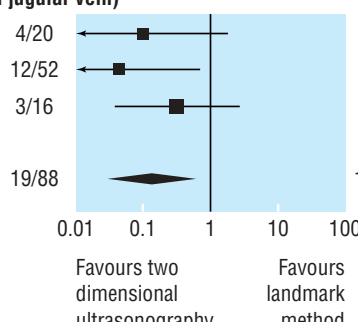

$26.30 .11(0.01$ to 1.94$)$

$27.40 .05(0.00$ to 0.79$)$

$46.30 .33(0.04$ to 2.87$)$

$100.00 .15(0.03$ to 0.64$)$

Fig 2 Risk of failure of catheter placement in studies of two dimensional ultrasound guidance compared with landmark method

7 Altman DG, Bland JM. Interaction revisited: the difference between two estimates. BMJ 2003;326:219

8 Randolph AG, Cook DJ, Gonzales CA, Pribble CG. Ultrasound guidance for placement of central venous catheters: a meta-analysis of the literature. Crit Care Med 1996;24:2053-8.

9 National Institute for Clinical Excellence. Guidance on the use of ultrasound locating devices for placing central venous catheters. London: NICE, 2002. [NICE Technology Appraisal No 49.]

(Accepted 18 June 2003) 